\title{
Pressure Effects on Superconducting and Magnetic Transitions in Layered Sodium Cobalt Oxides, $\mathrm{Na}_{x} \mathrm{CoO}_{2}$
}

\author{
Y. V. Sushko' ${ }^{1}$, O. B. Korneta ${ }^{1}$, S. O. Leontsev', \\ R. Jin ${ }^{2}$, B. C. Sales ${ }^{2}$, and D. Mandrus ${ }^{2}$ \\ 'Department of Physics and Astronomy, University of Kentucky, Lexington \\ KY 40506, USA \\ ${ }^{2}$ Condensed Matter Science Division, ORNL, Oak Ridge, TN 37831, USA
}

The results of dc magnetization measurements under hydrostatic (heliumgas) pressure are presented for an ambient pressure superconductor $\mathrm{Na}_{0.35} \mathrm{CoO}_{2} 1.4 \mathrm{D}_{2} \mathrm{O}$ and its precursor compound $\mathrm{Na}_{0.75} \mathrm{CoO}_{2}$ known to combine metallic conductivity with an unusual magnetic state below $\sim 22 \mathrm{~K}$. PACS numbers: 74.62.Fj, 74.70.-b, 75.20. En, 75.50 Ee, $75.30 \mathrm{Kz}$.

\section{INTRODUCTION}

The versatile family of sodium cobalt oxides emerged recently as a new target object for theoretical and experimental studies of various aspects of magnetism, superconductivity, and their interplay. The series $\mathrm{Na}_{\mathbf{x}} \mathrm{CoO}_{2}$ $(x<1)$ includes, besides a new superconductor ${ }^{1}, \mathrm{Na}_{0.3} \mathrm{CoO}_{2} \mathrm{yH}_{2} \mathrm{O}(\mathrm{y}=1.3)$ and its parent compound $\mathrm{Na}_{0.75} \mathrm{CoO}_{2}$, the material remarkable on its own for an antiferromagnetic order combined with metallic conductivity ${ }^{2-4}$, also such fascinating materials as the $x=2 / 3$ "Curie-Weiss metal" exhibiting abnormally large and spin-dependent thermopower ${ }^{5}$, and the $x=1 / 2$ compound, shown to posses an insulating low-temperature state with charge ordering on both the $\mathrm{CoO}_{2}$ and $\mathrm{Na}$ planes ${ }^{6}$.

It is known that both superconductivity and magnetism of $\mathrm{Na}_{\mathrm{x}} \mathrm{CoO}_{2}$ compounds originate within the $\mathrm{CoO}_{2}$ layers. Onset of a magnetically ordered state in $\mathrm{Na}_{0.75} \mathrm{CoO}_{2}$ is signaled by the phase transition observed at $\sim 22 \mathrm{~K}$ in various properties of the material. This transition was recently investigated in detail by Sales et al. ${ }^{4}$ on floating zone (FZ) grown single crystals. It was found that like in polycrystalline samples, the susceptibility above $50 \mathrm{~K}$ follows the $\chi=\chi_{0}+C /(T-\Theta)$ dependence with a negative Weiss temperature of about $200 \mathrm{~K}$. On cooling below $T_{\mathrm{N}}=22 \mathrm{~K}$ in small and moderate fields the susceptibility for both in-plane and out-of-plane orientations of magnetic field shows an abrupt increase. Magnetization curves at $1.8 \mathrm{~K}$ for both $H / / \mathbf{a}$ and $H / / \mathrm{c}$ directions show typical for ferromagnet hysteresis with remnant moment of $\sim 1 \times 10^{-4} \mu_{B}$ per Co atom, 
thus suggesting a weak ferromagnetism. Among the models proposed to explain magnetism in metallic sodium cobaltates, an SDW formation, initially suggested by Motohashi et al. ${ }^{2}$ and Sugiyama $e t a l{ }^{3}$, is favored by most of the current workers.

Measuring the pressure dependence of the magnetic ordering temperature can provide the direct insight into the ordering mechanism and also its relationship to the electronic structure since an applied pressure can change overlap between electron orbitals and thus cause a shift and/or split of the energy levels. However, despite that some pressure/volume dependent measurements on $\mathrm{Na}_{\mathrm{x}} \mathrm{CoO}_{2}$ were reported already ${ }^{7,8}$, we are still lacking pressure-dependent data on magnetic properties of $\mathrm{Na}_{\mathrm{x}} \mathrm{CoO}_{2}$. In this paper, the pressure dependence of the $22 \mathrm{~K}$ magnetic transition in the $\gamma$-phase $\mathrm{Na}_{\mathrm{x}} \mathrm{CoO}_{2} \mathrm{FZ}$ grown crystals ${ }^{4}$ with $x \sim 0.75$ and the superconducting $T_{c}$ of $\mathrm{Na}_{0.35} \mathrm{CoO}_{2} 1.4 \mathrm{D}_{2} \mathrm{O}$ polycrystalline sample ${ }^{9}$ are investigated by means of SQUID magnetometry under hydrostatic (helium-gas) pressure ${ }^{10}$.

\section{RESULTS AND DISCUSSION}

The temperature dependence of the magnetic moment along c-axis measured under two different values of applied pressures is presented in the left panel of Fig. 1. The data are shown in a narrow temperature interval in the vicinity of $T_{N}$ in order the details of the transition be clearly seen. The main effect of pressure is observed as a systematic shift of the transition curve to the higher temperatures. Increase of external pressure from 50 bar to $8.65 \mathrm{kbar}$, the highest pressure in our experiments, resulted in a shift of the transition onset point from $\sim 22.1 \mathrm{~K}$ to $\sim 24.3 \mathrm{~K}$, thus yielding a $10 \%$ growth of $T_{N}$. The values of $T_{N}$ for seven different pressures plotted against $P$ in the right figure allows us to summarize that an overall pressure dependence of the magnetic transition temperature of $\mathrm{Na}_{0.75} \mathrm{CoO}_{2}$ is linear, with the large positive baric coefficient $d T_{N} / d P=0.25 \mathrm{~K} / \mathrm{kbar}$.

The observed positive pressure effect on magnetic transition is quite unexpected for the $\mathrm{Na}_{0.75} \mathrm{CoO}_{2}$ in view that already several workers have concluded on the SDW-type ordering in this system. Indeed, it is well known, the chromium and organic Bechgaard salts $\mathrm{TMTSF}_{2} \mathrm{X}$ are among the textbook examples, that application of pressure has detrimental effect on an SDW state. Interpretation of this fact is rather straightforward - the SDW formation in a metal requires the presence of congruent (nested) parts on the Fermi surface (FS) and while a perfect nesting is a property of electronic band structure of 1-dimensional or quasi-one-dimensional systems with cylindrical FS, if the dimensionality (and the transverse dispersion)increases, the nesting property deteriorates due to cylinder wrapping. Since pressure is 
expected to suppress the anisotropy effects and it should reduce (and eventually suppress completely) the FS nesting, and this is exactly what happens in chromium, as well as $\mathrm{TMTSF}_{2} \mathrm{X}$ salts $\left(\mathrm{X}=\mathrm{AsF}_{6}, \mathrm{PF}_{6}\right)$.
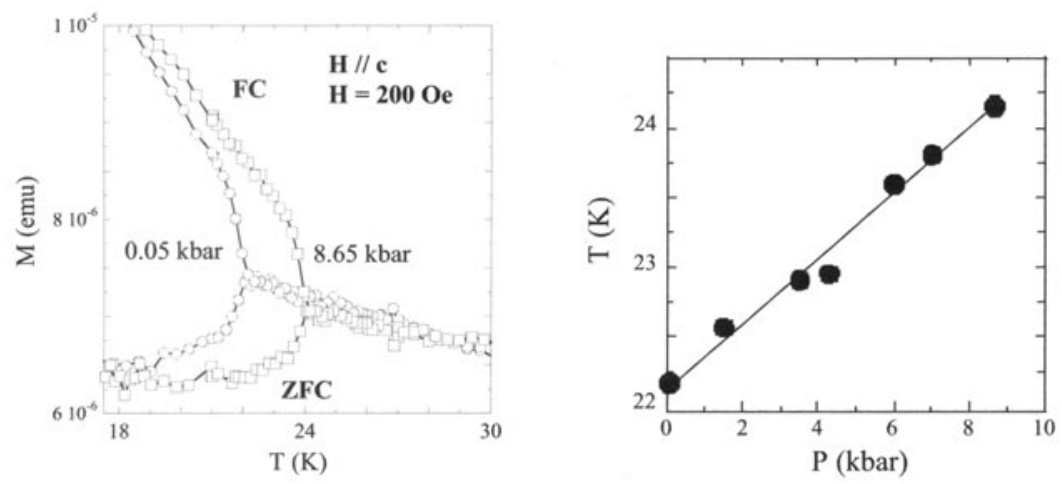

Fig. 1. Temperature dependence of magnetization of $\mathrm{Na}_{0.75} \mathrm{CoO}_{2}$ for field cooling (FC) and zero field cooling (ZFC) processes at two different pressures (left) and the overall (for the range $0-9 \mathrm{kbar}$ ) pressure dependence of the magnetic transition temperature (right).

Therefore, we conclude that a band antiferromagnetism scenario is not consistent with the detected experimentally positive pressure effect on $T_{N}$. An alternative interpretation could be a combination of itinerant and localized moment mechanisms, a scenario in which strongly anisotropic, layered character of $\mathrm{Na}_{0.75} \mathrm{CoO}_{2}$, with a rather suppressed interlayer transport (a value of 800 has been observed ${ }^{4}$ for the $\rho_{c} / \rho_{a b}$ ratio at $T=4 K$ ) plays a crucial role. Namely, we propose an A-type antiferromagnetism with the ferromagnetic alignment of the itinerant spins within the highly-conducting $\mathrm{CoO}_{2}$ planes and either superexchange- or Ruderman-Kittel-Kasuya-Yosida (RKKY) interaction- mediated antiferromagnetic inter-plane coupling. If superexchange is indeed important in $\mathrm{Na}_{0.75} \mathrm{CoO}_{2}$, the increase of $T_{N}$ under pressure is naturally ascribed to the compression of the Co-O-Co bond. Furthermore, since the RKKY exchange term $J(\mathbf{r})$ is distance dependent, the lattice compression also could lead to $T_{N}$ increasing with pressure.

The temperature dependence of the static susceptibility of superconducting compound $\mathrm{Na}_{0.35} \mathrm{CoO}_{2}{ }^{-1} \cdot \mathrm{D}_{2} \mathrm{O}$ measured at four different pressures is shown in Fig. 2. The right panel of the figure presents the $T_{c}$ vs. $P$ dependence. For comparison, the data of Lorenz et al. ${ }^{7}$, obtained on the polycrystalline sample of $\mathrm{Na}_{0.3} \mathrm{CoO}_{2} \cdot 1.3 \mathrm{H}_{2} \mathrm{O}$ by means of ac susceptibility measurements with the clamp cell technique are also shown. Although the magnitudes of the overall pressure shift of the superconducting critical 
temperature are rather close (compare our value of $-0.09 \mathrm{GPa}^{-1}$ with -0.07 $\mathrm{GPa}^{-1}$ reported by Lorenz), and the sign is negative, as expected (decreasing interlayer separation should ultimately destroy superconductivity), an important difference is noticeable. Namely, our data reveal that the $T_{c}$ varies linearly with $P$, whereas the Ref. 7 emphasizes strong nonlinearity in $T_{c}$ vs. $P$ relation. Such a discrepancy may reflect different sample quality and/or strong deviations from hydrostatic conditions in clamped-cell experiments.
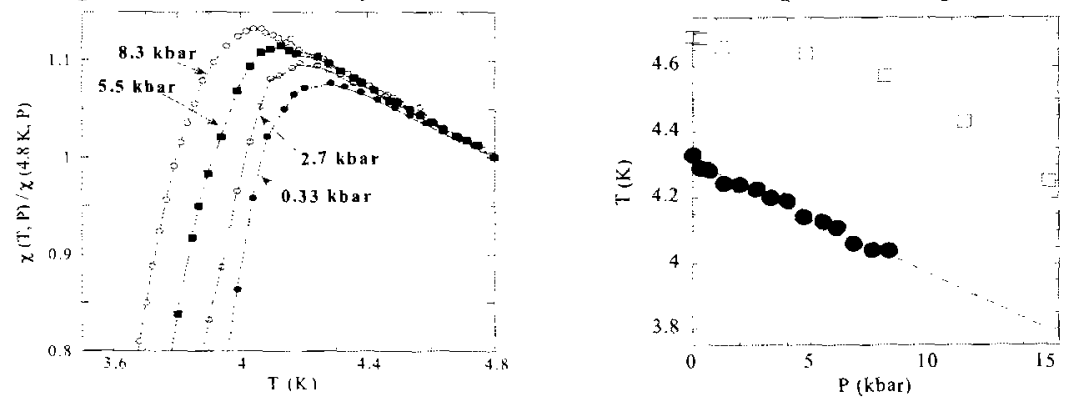

Fig. 2. Temperature dependence of magnetization of $\mathrm{Na}_{0.35} \mathrm{CoO}_{2} \cdot 1.4 \mathrm{D}_{2} \mathrm{O}$ for four different pressures (left). Right: $T_{c}$ vs. $P$ data of present work (circles) and the data of Ref. 7 (open squares) plotted on the same graph.

\section{ACKNOWLEDGEMENTS}

This work was supported in part by NSF (Grant No. DMR 0502706).

\section{REFERENCES}

1. K. Takada et al., Nature(London) 422, 53 (2003).

2. T. Motohashi et al., Phys. Rev. B 67, 064406 (2003).

3. J. Sugiyama et al., Phys. Rev. B 67, 214420 (2003).

4. B. C. Sales et al., Phys. Rev. B 70, 174419 (2004).

5. Y. Wang et al., Nature (London) 423, 425 (2003).

6. M. L. Foo et al., Phys. Rev. Lett. 92, 247001 (2004).

7. B. Lorenz et al., Phys. Rev. B 68, 132504 (2003).

8. S. Park et al., Phys. Rev. B 68, 180505 (R) (2003).

9. R. Jin et al., Phys. Rev. Lett. 91, 217001 (2003).

10. Y. V. Sushko et.al., Solid State Commun. 130, 341 (2004). 